\title{
Comparative study on virus shedding patterns in nasopharyngeal and fecal specimens of COVID-19 patients
}

\author{
Ning Zhang ${ }^{1 \dagger}$, Yuhuan Gong ${ }^{2 \dagger}$, Fanping Meng ${ }^{3}$, Yi Shi ${ }^{1}$, Jun Wang ${ }^{1}$, Panyong Mao $^{3}$, Xia Chuai ${ }^{*}$, \\ Yuhai $\mathrm{Bi}^{1,5,6^{*}}$, Penghui Yang ${ }^{3^{*}}$ \& Fusheng Wang ${ }^{3}$ \\ ${ }^{1}$ CAS Key Laboratory of Pathogenic Microbiology and Immunology, Institute of Microbiology, Center for Influenza Research and Early- \\ warning (CASCIRE), CAS-TWAS Center of Excellence for Emerging Infectious Diseases (CEEID), Chinese Academy of Sciences, Beijing \\ 100101, China; \\ ${ }^{2}$ Institutes of Physical Science and Information Technology, Anhui University, Hefei 230601, China; \\ ${ }^{3}$ The Fifth Medical Center of PLA General Hospital, National Clinical Research Center for Infectious Diseases, Beijing 100039, China; \\ ${ }^{4}$ Department of Pathogenic Biology, Hebei Medical University, Shijiazhuang 050017, China; \\ ${ }^{5}$ Shenzhen Key Laboratory of Pathogen and Immunity, Second Hospital Affiliated to Southern University of Science and Technology, Shenzhen \\ Third People's Hospital, Shenzhen 518112, China; \\ ${ }^{6}$ University of Chinese Academy of Sciences, Beijing 101408, China
}

Received May 24, 2020; accepted July 26, 2020; published online August 5, 2020

Citation: $\quad$ Zhang, N., Gong, Y., Meng, F., Shi, Y., Wang, J., Mao, P., Chuai, X., Bi, Y., Yang, P., and Wang, F. (2021). Comparative study on virus shedding patterns in nasopharyngeal and fecal specimens of COVID-19 patients. Sci China Life Sci 64, 486-488. https://doi.org/10.1007/s11427-020-1783-9

Dear Editor,

An acute viral pneumonia (COVID-19), caused by the novel coronavirus known as SARS-CoV-2, was first identified during December 2019 in China (Zhu et al., 2020). SARSCoV-2 was found to be highly transmissible in humans (Wang et al., 2020) and is now a pandemic spreading to over 140 countries and causing over 150,000 infections and 6,000 deaths as of March 15, 2020.

Diagnosis is critical for confirmation and treatment of COVID-19. Currently the primary criterion for diagnosis of COVID-19 is viral RNA detection for respiratory samples. Recently, two studies on virus loads in clinical samples reported that viral loads in nasal and throat swabs and sputum specimens peaked at 3-7 days after illness onset (d.a.o.) and virtually disappeared before 15 d.a.o. (Pan et al., 2020; Zou et al., 2020). Another study showed that the median duration

$\dagger$ Contributed equally to this work

*Corresponding authors (Yuhai Bi, email: beeyh@im.ac.cn (lead contact); Penghui Yang, email: ypenghuiamms@hotmail.com; Fusheng Wang, email: fswang302@163. com) of virus shedding in throat swabs was 20 d.a.o. in survivors and was detectable until death in non-survivors (Zhou et al., 2020). Additionally, live viruses have been isolated in the feces and urine samples of COVID-19 patients. However, the viral dynamics in these types of specimens has not yet been clearly elucidated, as well as comparative studies on virus shedding in the upper respiratory, intestinal, and urinary tracts.

From January 20 to February 23, 2020, a total of 23 patients were treated in a designated hospital in Beijing (11 were imported cases, 12 were secondary cases; two family clusters; 12 males, 11 females; the median age was 48.0 years (IQR 40.0 to 62.0 ); two were critically ill, the others were mild-to-moderate, all patients recovered except for one due to bacterial infection) (Table S1 in Supporting Information). Upper respiratory (nasal-throat mixed) swabs $(n=66)$, feces $(n=51)$, urine $(n=56)$, and plasma $(n=56)$ samples were collected for viral RNA detection by real-time RT-PCR (rRT-PCR) kit (Mabsky Biotech Co., Ltd.). The study was approved by the Ethics Committees of Chinese Academy of Sciences (APIMCAS2020057). Informed con- 
sent was obtained from all subjects for being included in the study, and all patient data were anonymized before study inclusion.

The plasma and urine samples were found to be negative, except for urine samples from two critically ill patients at the latest available detection point (16 or 21 d.a.o.). Conversely, virus was shed in respiratory swabs and fecal samples during the disease period (Figure 1A). Ten of 12 cases $(83.3 \%)$ were positive for fecal samples, while 14 of 21 cases $(66.7 \%)$ were positive for respiratory samples. In addition, all samples from one critically ill patient were negative until 21 d.a.o., when the fecal sample was positive. The median duration of virus shedding was 10.0 days (IQR 8.0 to 17.0 ) in nasal-throat mixed swabs, but was 22.0 days (IQR 15.5 to 23.5) for the feces (Figure 1A and B). The viral titers of nasal-throat swabs peaked at 6-9 d.a.o. and at 14-18 d.a.o. for fecal samples, and the mean virus titers at the peak were significantly higher for feces $\left(10^{6.3}\right.$ copies $\mathrm{mL}^{-1}$, mean 5,623 copies $\mathrm{mL}^{-1}$ ) than for respiratory samples $\left(10^{5.8}\right.$ copies $\mathrm{mL}^{-1}$, mean 2,535 copies $\mathrm{mL}^{-1}$ ) (Figure $1 \mathrm{~A}$ ). Notably, at 26 days after discharge, case 3 (the father of a family cluster) was detected to be positive again in the fecal sample, but appeared to be healthy and negative for the respiratory swabs. This family cluster includes the mother (case 1), father (case 3) and son (case 2) (Table S1 in Supporting Information). They all have travel history in Wuhan for four days. The mother firstly presented symptoms and was the first confirmed for COVID-19. Six days later the father and son displayed symptoms and were admitted to hospital. For this family cluster, we found that the fecal samples were continuously positive until discharge, especially for the mother and son, while their respiratory samples became negative after one weeks' treatment in hospital (Figure 1C). Comparative analysis on the imported
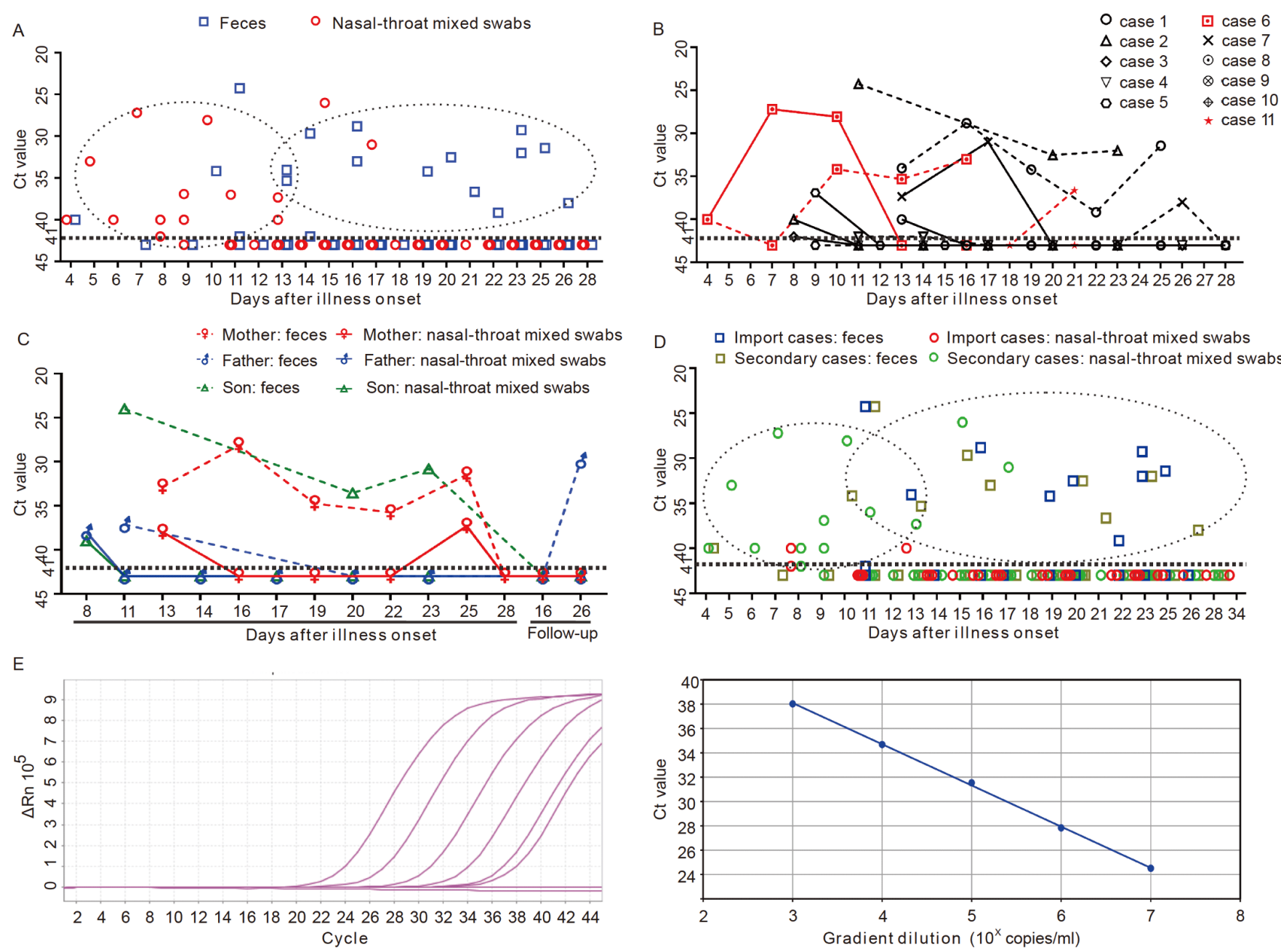

Figure 1 Virus dynamics in nasopharyngeal and fecal specimens of COVID-19 cases. A, The nasal-throat mixed swabs $(n=66)$ and fecal samples $(n=51)$ of all 23 cases were collected and detected by rRT-PCR. B, Sequential nasal-throat mixed swab (solid line) and fecal samples (dotted line) were collected in 11 cases and used for virus detection. Two critically ill cases were colored in red. The other nine cases with mild-to-moderate symptoms were colored in black. C, Virus detection of a family cluster includes the mother, father, and son. D, Comparative analysis on virus titers between the imported (red and blue) and secondary cases (green and cyan). E, The standard curve, produced by the pseudotyped virus with SARS-CoV-2 ORFlab gene, for calculation of viral RNA copy numbers. The regression curve is $y=-3.5691 x+47.127\left(R^{2}=0.9993\right)$, where $x$ represents the gradient dilution factor, $y$ represents $C_{\mathrm{t}}$ values. 
and secondary cases showed that the fecal samples were generally positive from 11 to 25 d.a.o. in both groups. However, the respiratory samples were positive with higher virus titer in the early stages of infection for the secondary cases, which was not seen in the imported cases (Figure 1D).

Therefore, we found that the period of virus shedding was significantly different between the upper respiratory and fecal samples. A longer virus shedding period was found in the fecal samples compared to the upper respiratory samples, although the viral RNAs in the latter were generally detectable earlier than in the former. In addition, one recent study reported that an asymptomatic case was positive in the rectal swabs but negative in the nasopharyngeal swabs for a period as long as 42 days (Jiang et al., 2020). Our data suggest that detection of respiratory samples together with fecal samples should be used for diagnosis of COVID-19, especially for monitoring the relapse of discharged patients, the later stages of infection, and latent or asymptomatic infection, which will be more effective than detection with only one sample type. Furthermore, live virus has been isolated in fecal samples (Xiao et al., 2020), suggesting a potential fecal-oral transmission route for COVID-19. Hence, this comparative study on viral shedding between the upper respiratory and fecal samples provides a new clue for the diagnosis and prevention of COVID-19.

Compliance and ethics The author(s) declare that they have no conflict of interest.
Acknowledgements This work was supported by the Strategic Priority Research Program of the Chinese Academy of Sciences (CAS) (XDB29010102 and XDA19090118), the National Natural Science Foundation of China (NSFC) (32041010), and Hebei Key Research and Development Program (20277704D). Y.B. is supported by the NSFC Outstanding Young Scholars (31822055) and Youth Innovation Promotion Association of CAS (2017122).

\section{References}

Jiang, X., Luo, M., Zou, Z., Wang, X., Chen, C., and Qiu, J. (2020). Asymptomatic SARS-CoV-2 infected case with viral detection positive in stool but negative in nasopharyngeal samples lasts for 42 days. J Med Virol jmv.25941.

Pan, Y., Zhang, D., Yang, P., Poon, L.L.M., and Wang, Q. (2020). Viral load of SARS-CoV-2 in clinical samples. Lancet Infect Dis 20, 411-412.

Wang, C., Horby, P.W., Hayden, F.G., and Gao, G.F. (2020). A novel coronavirus outbreak of global health concern. Lancet 395, 470-473.

World Health Organization. (2020). Coronavirus disease (COVID-19) outbreak. https://www.who.int/emergencies/diseases/novel-coronavirus-2019.

Xiao, F., Sun, J., Xu, Y., Li, F., Huang, X., Li, H., Zhao, J., Huang, J., and Zhao, J. (2020). Infectious SARS-CoV-2 in feces of patient with severe COVID-19. Emerg Infect Dis 26, 1920-1922.

Zhou, F., Yu, T., Du, R., Fan, G., Liu, Y., Liu, Z., Xiang, J., Wang, Y., Song, B., Gu, X., et al. (2020). Clinical course and risk factors for mortality of adult inpatients with COVID-19 in Wuhan, China: a retrospective cohort study. Lancet 395, 1054-1062.

Zhu, N., Zhang, D., Wang, W., Li, X., Yang, B., Song, J., Zhao, X., Huang, B., Shi, W., Lu, R., et al. (2020). A novel coronavirus from patients with pneumonia in China, 2019. N Engl J Med 382, 727-733.

Zou, L., Ruan, F., Huang, M., Liang, L., Huang, H., Hong, Z., Yu, J., Kang, M., Song, Y., Xia, J., et al. (2020). SARS-CoV-2 viral load in upper respiratory specimens of infected patients. N Engl J Med 382, $1177-$ 1179.

\section{SUPPORTING INFORMATION}

The supporting information is available online at https://doi.org/10.1007/s11427-020-1783-9. The supporting materials are published as submitted, without typesetting or editing. The responsibility for scientific accuracy and content remains entirely with the authors. 